TEPP RAPPORT DE RECHERCHE

\title{
Dépréciation du capital humain et formation continue au cours du cycle de vie : Quelle dynamique des externalités sociales?
}

ARNAUD CHERON, ANTHONY TERRIAU 
Dépréciation du capital humain et formation continue au cours du cycle de vie:

Quelle dynamique des externalités sociales?

Arnaud Chéron*

Université du Maine (GAINS) et EDHEC Business School

Anthony Terriau ${ }^{\dagger}$

Université du Maine (GAINS)

Décembre 2014

*acheron@univ-lemans.fr

†anthony.terriau@univ-lemans.fr 


\begin{abstract}
Dans cette étude, nous présentons un cadre théorique permettant d'analyser les interactions entre investissement en capital humain, turbulence économique et cycle de vie afin d'examiner la dynamique des externalités sociales liées à la formation générale. L'existence de telles externalités conduit à exclure certains travailleurs du système de formation professionnelle, à tort du point de vue de ce qui serait socialement souhaitable. Il convient donc de proposer une politique de soutien à la formation ciblée vers certains types de travailleurs. La principale contribution de ce papier est en effet de positionner cette problématique des externalités sociales de la formation continue dans la perspective du cycle de vie du travailleur, et de souligner qu'il serait nécessaire de mettre en place une politique d'incitation à la formation dépendante de l'âge. Nous montrons en effet que les employeurs tendent à accroître trop tôt dans le cycle de vie des travailleurs leur critère d'accès à la formation continue relativement à ce que suggérerait de faire la prise en compte des externalités sociales. En revanche, en toute fin de carrière (à l'approche de la retraite) le rendement social converge vers le rendement privé de la formation et tend vers zéro. Au bilan, ceci plaide donc pour une politique incitative non monotone avec l'âge, croissante jusqu'à un certain âge, puis décroissante ensuite.
\end{abstract}




\section{Introduction}

Le diagnostic du non emploi des seniors, particulièrement élevé en France, est bien établi: les taux d'emploi des 55-59 ans et 60-64 ans équivalent respectivement à $67,5 \%$ et $23,3 \%$, contre 80,8\% par exemple pour les 25-49 ans. Comme l'ont déjà souligné Chéron, Hairault et Langot [2011, 2013], les effets d'horizon associés à un âge de retraite, relativement plus faible en France par rapport à la moyenne OCDE, sont en partie responsables de ce diagnostic. Depuis 2003, les réformes successives du système de retraite français ${ }^{1}$ ont conduit à un relèvement de l'âge effectif de départ qui, à l'inverse, a favorisé l'emploi des seniors, avec un relèvement de plus de 12 points du taux d'emploi des 55-59 ans, et supérieur à 8 points pour les 60-64 ans. De façon complémentaire, la problématique générale de cette étude s'inscrit dans la détermination des politiques optimales de l'emploi susceptibles de requérir un design spécifique selon l'âge, et donc notamment à destination des seniors. Plus précisément, cette étude propose d'examiner la question des politiques de soutien à la formation professionnelle continue.

Les seniors (comme les moins qualifiés) ont relativement moins accès à la formation professionnelle : selon les données de l'INSEE (enquête sur la formation des adultes 2012), le taux annuel d'accès à une formation professionnelle (quelle que soit sa durée) équivaut à $20 \%$ pour les $55-64$ ans, contre près de $40 \%$ pour les $25-44$ ans $^{2}$. Les évaluations empiriques fournies par Lignon [2014], sur la base du modèle de microsimulation GAMEO des trajectoires professionnelles, estimé conjointement sur les données de l'Enquête Emploi 2003-2010 de l'INSEE et son complément "formation continue" 2006 confirment également ce diagnostic (Figure 1): le pic de l'accès à la formation professionnelle continue se situe entre 30 et 45 ans, et on note un décrochage très marqué au-delà de 50 ans, et ce, quels que soient le sexe et le niveau de diplôme des travailleurs.

Ce constat général soulève deux interrogations. La première concerne les raisons de ce décrochage en fin de carrière. Les travaux de Chéron [2007] ont notamment déjà souligné que le déclin de l'accès à la formation continue des seniors pourrait s'expliquer par l'effet d'horizon de la retraite, impliquant que la rentabilité intertemporelle des dépenses de formation se réduit à mesure que l'âge de la retraite du salarié se rapproche. La deuxième, qui constitue le coeur de la problématique et l'originalité de la présente étude, est d'ordre normatif: fautil nécessairement aller contre ce déclin de l'accès à la formation professionnelle avec l'âge ? Jusqu'à quel âge soutenir la formation continue? Sous quelle forme? Y a-t-il une classe d'âge "prioritaire"? Répondre à ces questions rend nécessaire une analyse en termes d'efficacité économique, au sens de la prise en considération des externalités sociales, éventuellement dépendantes de l'âge.

\footnotetext{
${ }^{1}$ On pense notamment à l'augmentation de l'âge minimum légal de retraite et à la libéralisation du dispositif de cumul emploi-retraite.

${ }^{2}$ Sur l'ensemble des tranches d'âge, le taux d'accès moyen pour les ouvriers est de 37\%, $43 \%$ pour les employés, $61 \%$ pour les professions intermédiaires, et $68 \%$ pour les cadres.
} 
Figure 1: Taux d'accès à la formation continue par âge et par diplôme
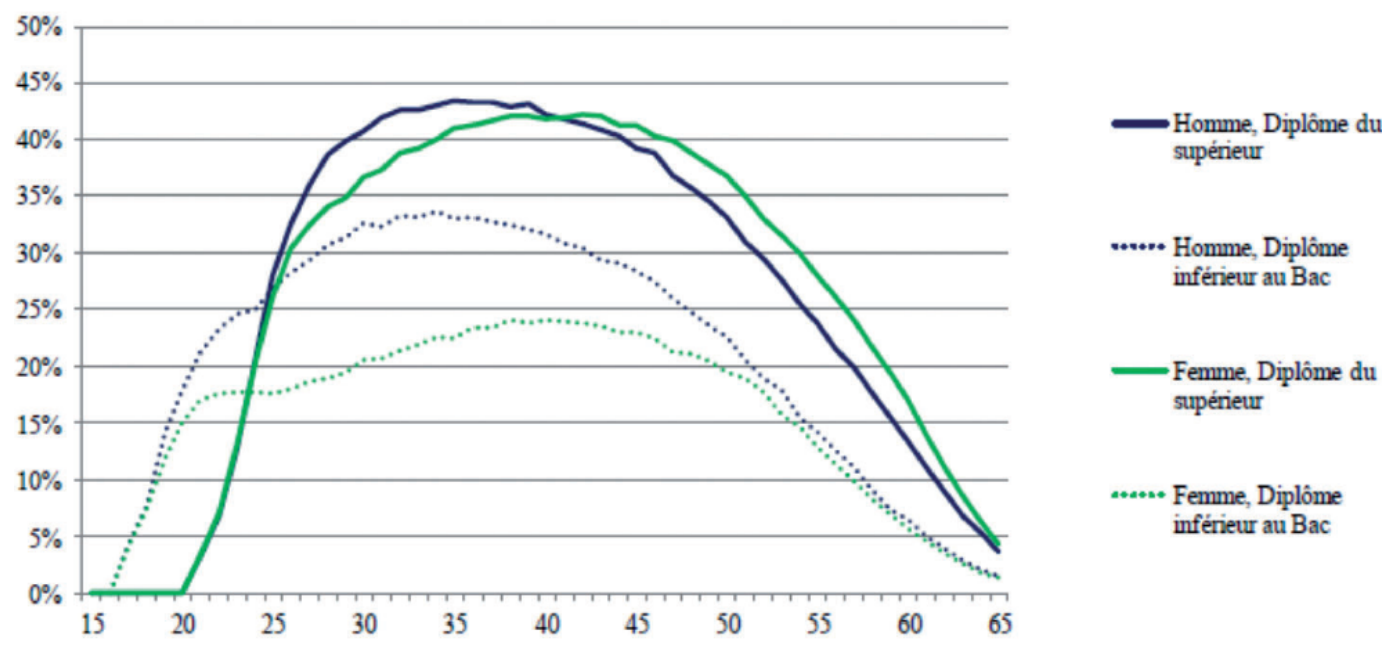

Source : Modèle GAMEO, module formation continue (Lignon [2014])

Le rendement privé (en termes de productivité) de la formation continue des salariés par les employeurs - qui constituent le premier financeur de la formation continue des actifs occupés ${ }^{3}$ - est typiquement inférieur à son rendement social, qui intègre son impact sur la productivité dans des emplois ultérieurs mais aussi les bienfaits en termes d'employabilité si le travailleur se trouve ultérieurement concerné par le chômage. Nous le verrons, ces externalités impliquent qu'il existe un écart entre les décisions des entreprises et les décisions optimales socialement, cet écart évoluant selon l'âge des travailleurs. À cette fin, nous sommes amenés à développer une analyse structurelle explicitant les choix de formation professionnelle continue en fonction de l'âge et intégrant la diversité des externalités induites au niveau social par ces dépenses de formation. Nous proposerons ainsi différentes évaluations quantitatives (simulations) basées sur un modèle frictionnel du marché du travail où la formation est un moyen d'accroître le capital humain des travailleurs, mais où les périodes de chômage constituent un facteur de dégradation éventuelle des compétences accumulées.

Indépendamment de la question de l'âge, dans le contexte d'un marché du travail frictionnel caractérisé par des délais d'embauche et un mode de fixation des salaires non concurrentiel, la sous-optimalité (ou encore l'inefficacité économique) des décisions de formation en capital humain général est un résultat bien établi. Les employeurs investissent insuffisamment dans des compétences et savoirs transférables de leurs salariés, au regard de ce qui serait socialement souhaitable. L'écart entre le rendement privé et le rendement collectif des dépenses de formation professionnelle continue résulte notamment de l'existence de deux types d'externalités, sur lesquelles il convient de revenir.

\footnotetext{
${ }^{3}$ D'après les données de la DARES, les entreprises supportent plus de $85 \%$ des dépenses de formation professionnelle continue à destination des actifs occupés du secteur privé.
} 
À la fin des années quatre-vingt-dix, les travaux de Daron Acemoglu ont proposé de réévaluer l'approche traditionnelle des investissements en capital humain dans le cadre d'un marché du travail frictionnel. Jusque-là, les travaux de Becker [1964] ${ }^{4}$ suggéraient que, sur un marché du travail concurrentiel (caractérisé notamment par l'absence de chômage), les investissements en capital humain général étaient financés de manière optimale par les travailleurs, au travers de baisses de salaires (ou de modérations salariales). Partant néanmoins du constat que les employeurs sont disposés à contribuer aux dépenses de formation professionnelle, ces auteurs ont souligné que, du fait d'une mobilité imparfaite des salariés entre les emplois, et d'un pouvoir de négociation salariale significatif des employeurs, ces derniers sont disposés à engager des dépenses en formation générale ${ }^{5}$, même s'ils ne sont en mesure d'en capter qu'une partie du rendement. En effet, ce rendement en termes de productivité dépasse le seul cadre de l'entreprise formatrice, dès lors qu'il s'agit de compétences transférables. Le travailleur est susceptible de mobiliser les savoirs acquis dans des emplois ultérieurs. Acemoglu et Pischke [1999] font ainsi référence à l'existence d'externalités de débauchage (poaching externality), qui rend nécessaire l'intervention de l'État.

Si les entreprises sont bel et bien disposées à dépenser pour la formation professionnelle continue de leurs salariés, y compris pour de la formation générale, elles ne le font donc pas suffisamment au regard du véritable rendement social de ces dépenses en termes de productivité, car seuls les travailleurs intègrent la valorisation du capital humain dans les emplois ultérieurs. Par conséquent, il en résulte un sous-investissement des entreprises dans la formation professionnelle de leurs salariés. On comprend alors que le pouvoir de négociation des travailleurs, qui financent indirectement leur formation via des salaires moindres, jouent un rôle crucial : plus il est important, plus les travailleurs sont disposés à contribuer au financement de leur formation, alors même qu'eux seuls internalisent le rendement social de ces investissements. A mesure que le pouvoir de négociation des salariés s'élève, il apparaît ainsi que les dépenses de formation s'approchent de leur contrepartie optimale.

Les travaux de Belan et Chéron [2011, 2014] (BC) mettent pour leur part en évidence l'existence d'une source additionnelle d'externalités sociales liées aux dépenses de formation professionnelle continue. Ceci s'inscrit dans une approche où les répercussions de la dépréciation du capital humain général pendant les épisodes de chômage sont étudiées, notamment en matière de (sous)optimalité des investissements en formation. ${ }^{6}$

\footnotetext{
${ }^{4}$ Becker distingue les investissements en capital humain spécifique qui accroissent la productivité du travailleur dans l'entreprise formatrice, et les investissements en capital humain général qui augmentent la productivité de l'agent au sein de n'importe quelle firme. Dans ce cadre, les firmes n'ont pas d'incitation à investir en capital humain général dans la mesure où les travailleurs peuvent capturer l'ensemble des gains issus de leur formation en menaçant de quitter l'entreprise formatrice pour un salaire plus élevé.

${ }^{5}$ L'OCDE [2003] estime que deux formations sur trois ont un contenu général.

${ }^{6}$ Dans une économie caractérisée par un développement des savoirs et une accélération du progrès technologique, Ljungqvist et Sargent [1998, 2004, 2007] ont montré que la hausse de la turbulence économique le risque pour un travailleur licencié de subir une dépréciation de son capital humain durant un épisode de chômage - combinée à un système d'assurance chômage généreux était à l'origine de la hausse du chômage en Europe dans les années 1980 et 1990. Belan et Chéron (2014) étudient l'impact de la turbulence économique sur les décisions de formation des entreprises et sur la politique optimale de subvention à la formation.
} 
Pour contrecarrer les effets de l'obsolescence éventuelle des compétences induites par le chômage, les entreprises ont la possibilité d'investir dans la formation professionnelle des travailleurs qu'elles embauchent afin de remettre à jour le capital humain du chômeur si le besoin s'en fait sentir. Dans ce contexte, Belan et Chéron soulignent que, si les individus ne bénéficiant pas de formation professionnelle sont confrontés à des durées de chômage relativement plus longues, le refus par les employeurs de former certains travailleurs est à l'origine d'une externalité sociale négative : pendant l'épisode de chômage, d'une durée relativement rallongée, le travailleur a un niveau de production "domestique" inférieur à sa production marchande potentielle, ce qui "coûte" à la société. Belan et Chéron montrent que cette forme d'externalité de chômage se combine à celle de débauchage, de sorte que certains travailleurs sont exclus de la formation professionnelle continue, à tort du point de vue de ce qui serait socialement souhaitable. A cet égard, il est donc légitime de réfléchir à un système conduisant les entreprises à investir dans la formation professionnelle de ces travailleurs, avec des modalités sur lesquelles nous reviendrons.

Nous proposons ici d'étendre cette approche en la plaçant dans un cadre de cycle de vie afin d'analyser les interactions entre les investissements en formation, la turbulence économique et l'âge des travailleurs. Dans ce cadre, nous développons un modèle frictionnel du marché du travail où la formation est un moyen d'accroître le capital humain général des travailleurs, mais où les périodes de chômage constituent un facteur de dégradation éventuelles des compétences accumulées. Il en ressort deux principaux résultats: (i) en toute fin de carrière (à l'approche de la retraite) le rendement social converge vers le rendement privé de la formation et tend vers zéro; (ii) les employeurs tendent à accroître trop tôt dans le cycle de vie des travailleurs leur critère d'accès à la formation continue relativement à ce que suggérerait de faire la prise en compte des externalités sociales. Par conséquent, il existe une tranche d'âge durant lequel il semble souhaitable de renforcer les incitations à la formation continue; cette tranche d'âge renvoie à ce laps de temps durant lequel les employeurs réduisent déjà l'accès à la formation continue alors que du point de vue du planificateur il reste optimal de proposer un accès similaire à celui offert aux plus jeunes.

$\mathrm{Au}$ travers d'une illustration quantitative nous proposons ensuite un faisceau d'éléments suggérant qu'il serait souhaitable de mettre oeuvre des incitations financières à destination des entreprises (conditionnellement à l'embauche des chômeurs) afin qu'elles forment prioritairement les travailleurs typiquement âgés de 50 à 57 ans. En revanche, au delà de 57 ans, c'est à dire à moins de 5 ans de la retraite, il ne semble plus souhaitable de maintenir un soutien à la formation continue.

Dans une première partie, nous présentons le cadre théorique et nous déterminons la politique de formation en fonction de l'âge des travailleurs à l'équilibre et à l'optimum. En comparant les décisions des entreprises à celle d'un planificateur social, nous montrons qu'il peut être souhaitable de subventionner la formation professionnelle sur certaines classes d'âge. Dans une seconde partie, nous présentons les simulations de notre modèle qui suggèrent de mettre en oeuvre une politique ciblée en faveur des 50-57 ans, et de ne pas soutenir la formation professionnelle au delà de cet âge. 


\section{Le cadre théorique de référence}

On considère un modèle à la Belan-Chéron [2014] (BC) étendu pour rendre compte de la dynamique par âge de l'accès à la formation. Chaque travailleur est caractérisé par un niveau d'aptitude $a$ qui détermine sa productivité en emploi. La politique de formation professionnelle continue revient à définir un seuil minimal d'aptitude ouvrant accès à une formation, qui consiste en "une mise à jour" des connaissances, postérieure à l'embauche, et permettant un supplément de productivité $\Delta a$ durant toute la durée de l'emploi. Cette formation engendre un coût, $\gamma_{f}$, supposé payé une fois pour toute à l'embauche. Pendant les éventuelles périodes de chômage, cette mise à jour est susceptible de se déprécier, traduisant une obsolescence des compétences acquises. Le seuil d'aptitude au-dessus duquel le travailleur a accès à la formation, noté $\tilde{a}_{t}$, évolue au cours du cycle de vie. L'analyse vise à caractériser les niveaux et la dynamique de ce seuil, d'une part résultant des choix des employeurs, et d'autre part défini optimalement en raisonnant sur la valeur sociale des dépenses de formation professionnelle continue.

\subsection{L'environnement économique}

Comme dans BC, les travailleurs se distinguent par leur type $j \in\{0 ; 1 ; 2\}$ qui dépend de leur niveau d'aptitude (diplôme), noté $a$, et de l'état de leurs connaissances:

- type 0 : individus dont l'aptitude est trop faible pour qu'une entreprise juge rentable de les former (individus d'aptitude $a<\tilde{a}^{t}$ )

- type 1 : individus dont l'aptitude est suffisante pour être éligible à une formation et dont les connaissances ont été déclassées (individus d'aptitude $a \geq \tilde{a}^{t}$ et de productivité pré-formation égale à $a$ )

- type 2 : individus ayant déjà été formés et qui n'ont pas subi de dépréciation de leur capital humain acquis au cours de leur formation en entreprise (individus d'aptitude $a$ et de productivité égale à $[1+\Delta] a)$

La probabilité qu'un chômeur d'âge $t$ soit employé à la période $t+1$ est supposée conditionnée par son aptitude et l'état de ses connaissances, de sorte que :

- $p_{0, t}$ pour les individus dont les connaissances sont dépréciées (type 0 ou 1) et ayant un niveau d'aptitude $a<\tilde{a}^{t+1}$ trop faible pour être formé à la période suivante en cas d'embauche.

- $p_{t}$ pour les individus de type 2 ou de type 1 et un niveau d'aptitude suffisant pour être formé en cas d'embauche à la période suivante $\left(a \geq \tilde{a}^{t+1}\right)$.

Chaque individu entre sur le marché du travail à l'âge $t=0$ en tant que chômeur et le quitte à un âge déterministe noté $T$, interprété comme l'âge de départ à la retraite. Le modèle est en temps discret et on suppose qu'à chaque période la génération de travailleurs la plus ancienne se retire du marché du travail et est remplacée par une nouvelle génération de même 
taille, de sorte que la population sur le marché du travail reste constante. Par conséquent, les travailleurs se distinguent par leur niveau d'aptitude, l'état de leurs connaissances (à jour ou non) et par la distance qui les sépare de la retraite.

En toute généralité, les individus recrutés à la période $t$ ne deviennent productifs qu'à la période suivante. La firme décide alors de former le travailleur nouvellement embauché si son aptitude est telle que l'espérance des gains issus de la formation permet, au minimum, de couvrir son coût. On suppose que tous les individus disposent d'un stock de capital humain dont une partie peut se déprécier au cours d'un épisode de chômage avec une probabilité $\pi$, c'est le phénomène de turbulence économique mis en évidence par Ljungqvist et Sargent [1998, 2004, 2007]. Autrement dit, certaines compétences acquises au cours de la formation initiale ou par le biais d'une formation en entreprise peuvent devenir obsolètes dès lors que l'individu n'est pas en mesure de les mettre en pratique en situation de travail.

\subsection{Définition des espérances de gains intertemporels sur le marché du travail}

Soient $w$ le salaire, $\delta$ la probabilité de destruction des emplois, $\beta$ le facteur d'escompte psychologique, et $b$ la production domestique en cas de chômage. On détermine les fonctions de gains intertemporels au cours du cycle de vie sur le marché du travail, pour les employés, notées $E_{j}^{t}(a)$, et pour les chômeurs, notés $U_{j}^{t}(a)$, de la manière suivante, $\forall t<T-1:^{7}$

type $0\left(\forall a<\tilde{a}^{t}\right)$ :

$E_{0}^{t}(a)=w_{0}^{t}(a)+\beta\left\{(1-\delta) E_{0}^{t+1}(a)+\delta U_{0}^{t+1}(a)\right\}$

$U_{0}^{t}(a)=b+\beta\left\{p_{0, t} E_{0}^{t+1}(a)+\left(1-p_{0, t}\right) U_{0}^{t+1}(a)\right\}$

type 1:

$E_{1}^{t}(a)=w_{1}^{t}(a)+\beta\left\{(1-\delta) E_{2}^{t+1}(a)+\delta U_{2}^{t+1}(a)\right\} \quad, \forall a \geq \tilde{a}^{t}$

$U_{1}^{t}(a)=b+\beta \begin{cases}p_{0, t} E_{0}^{t+1}(a)+\left(1-p_{0, t}\right) U_{0}^{t+1}(a) & , \forall a \in\left[\tilde{a}^{t} ; \tilde{a}^{t+1}[\right. \\ p_{t} E_{1}^{t+1}(a)+\left(1-p_{t}\right) U_{1}^{t+1}(a) & , \forall a \geq \tilde{a}^{t+1}\end{cases}$

type 2:

$E_{2}^{t}(a)=w_{2}^{t}(a)+\beta\left\{(1-\delta) E_{2}^{t+1}(a)+\delta U_{2}^{t+1}(a) \quad, \forall a\right.$

$U_{2}^{t}(a)=b+\beta \begin{cases}p_{t} E_{2}^{t+1}(a)+\left(1-p_{t}\right)(1-\pi) U_{2}^{t+1}(a)+\left(1-p_{t}\right) \pi U_{0}^{t+1}(a) & , \forall a<\tilde{a}^{t+1} \\ p_{t} E_{2}^{t+1}(a)+\left(1-p_{t}\right)(1-\pi) U_{2}^{t+1}(a)+\left(1-p_{t}\right) \pi U_{1}^{t+1}(a) & , \forall a \geq \tilde{a}^{t+1}\end{cases}$

\footnotetext{
${ }^{7}$ En T-1, on fait l'hypothèse que tous les individus sont certains de percevoir à la période suivante une pension de retraite universelle d'un montant unique, notée $R$.
} 
De même, du point de vue des employeurs on peut définir les fonctions-valeur des postes de travail, $J_{j}^{t}(a)$, de la façon suivante, $\forall t<T-1:^{8}$

$$
\begin{aligned}
& J_{0}^{t}(a)=a-w_{0}^{t}(a)+\beta(1-\delta) J_{0}^{t+1}(a) \\
& J_{1}^{t}(a)=(1+\Delta) a-w_{1}^{t}(a)+\beta(1-\delta) J_{2}^{t+1}(a) \\
& J_{2}^{t}(a)=(1+\Delta) a-w_{2}^{t}(a)+\beta(1-\delta) J_{2}^{t+1}(a)
\end{aligned}
$$

Les salaires sont pour leur part solution d'un jeu de négociation à la Nash, satisfaisant les règles de partage suivantes (cf. Appendice B):

$$
\begin{aligned}
(1-\alpha)\left[E_{0}^{t}(a)-U_{0}^{t}(a)\right] & =\alpha J_{0}^{t}(a) \\
(1-\alpha)\left[E_{1}^{t}(a)-U_{1}^{t}(a)\right] & =\alpha\left[J_{1}^{t}(a)-\gamma_{f}\right] \\
(1-\alpha)\left[E_{2}^{t}(a)-U_{2}^{t}(a)\right] & =\alpha J_{2}^{t}(a)
\end{aligned}
$$

avec $\alpha$ le pouvoir de négociation des travailleurs.

On peut alors caractériser les équations de salaires, selon le type d'individu et $\forall t<T-1$, de la façon suivante (voir l'annexe B pour le détail de la résolution):

- Individus de type 0 , non éligibles à la formation $\left(a<\tilde{a}^{t}\right)$ :

$$
w_{0}^{t}(a)=\alpha a+(1-\alpha) b+\alpha \beta p_{0}^{t}(a) J_{0}^{t+1}(a)
$$

- Individus de type 1 , éligibles à la formation pour la dernière fois $\left(\tilde{a}^{t}<a<\tilde{a}^{t+1}\right)$ :

$$
w_{1}^{t}(a)=\alpha\left[(1+\Delta) a-\gamma_{f}\right]+(1-\alpha) b+\alpha \beta p_{0}^{t}(a) J_{0}^{t+1}(a)-\beta(1-\alpha)\left[U_{2}^{t+1}(a)-U_{0}^{t+1}(a)\right]
$$

- Individus de type 2, ayant des connaissances à jour

$$
w_{2}^{t}(a)=\alpha(1+\Delta) a+(1-\alpha) b+\alpha \beta p^{t}(a) J_{2}^{t+1}(a)-\beta \pi(1-\alpha)\left[1-p^{t}(a)\right]\left[U_{2}^{t+1}(a)-U_{0}^{t+1}(a)\right]
$$

On notera en particulier que la valeur relative de la formation continue en cas d'épisode ultérieur de chômage, caractérisée par $U_{2}^{t+1}(a)-U_{0}^{t+1}(a)$, tend à réduire le salaire de réservation des individus en position d'être formés (de types 1 et 2 ), contribuant de ce fait à réduire les salaires négociés, $w_{1}^{t}(a)$ et $w_{2}^{t}(a)$.

\subsection{Caractérisation des décisions de formation par les employeurs}

La politique de formation des entreprises consiste à choisir un seuil d'aptitude critique, noté $\tilde{a}^{t} \forall t$, en dessous duquel le coût de la formation devient supérieur au rendement intertemporel espéré; il vérifie donc: $J_{1}^{t}\left(\tilde{a}^{t}\right)-\gamma_{f}=J_{0}^{t}\left(\tilde{a}^{t}\right)$.

\footnotetext{
${ }^{8}$ On a également $J_{0}^{T-1}(a)=a$ et $J_{1}^{T-1}(a)=J_{2}^{T-1}(a)=(1+\Delta) a$.
} 
Il est alors possible de montrer que: (cf. Annexe C)

$\Delta \tilde{a}^{T-1}=\gamma_{f}$

$\Delta \tilde{a}^{T-2}=\frac{\gamma_{f}}{\sum_{i=0}^{1} \beta^{i}(1-\delta)^{i}}$

$\Delta \tilde{a}^{t}=\frac{\gamma_{f}-\left\{\sum_{i=0}^{T-3-t} \beta^{i+1} \delta(1-\delta)^{i}\left[U_{2}^{t+1+i}\left(\tilde{a}^{t}\right)-U_{0}^{t+1+i}\left(\tilde{a}^{t}\right)\right]\right\}}{\sum_{i=0}^{T-1-t} \beta^{i}(1-\delta)^{i}}, \forall t \leq T-3$

- En $T-1$, l'arbitrage est statique et le seuil d'aptitude est tel que le gain de productivité issu de la formation couvre son coût instantanné.

- En $T-2$, le facteur d'actualisation apparaît, puisque le gain de productivité intertemporel peut intervenir sur deux périodes.

- $\forall t \leq T-3$, le seuil devient dépendant de l'écart de valeur du chômage (via la négociation salariale), l'individu ayant alors une certaine probabilité de connaitre une période de chômage puis d'être réembauché. Il aura alors la possibilité de réutiliser les compétences acquises au cours d'une formation professionnelle auprès d'un nouvel employeur sous réserve qu'elles ne se déprécient pas; ceci renvoie à l'écart de valeur du chômage selon que l'individu est de type 0 ou de type 2 .

L'annexe C propose une résolution explicite de ces seuils pour $t \geq T-4$, explicitant $U_{2}^{t+1}\left(\tilde{a}^{t}\right)-$ $U_{0}^{t+1}\left(\tilde{a}^{t}\right)$ en fonction des différentes probabilités de transition et leur combinaison au cours du cycle de vie.

\subsection{Caractérisation des décisions optimales de formation}

A l'optimum, on raisonne sur les valeurs sociales du travailleur selon son âge, son niveau d'aptitude et son statut 0,1 ou 2 vis à vis de la formation:

- La valeur sociale d'un travailleur occupant un emploi, et dont les compétences professionnelles sont maximales du fait d'avoir bénéficié d'une formation professionnelle qui ne s'est pas dépréciée:

$$
\tilde{Y}_{t}(a)=(1+\Delta) a+\beta\left[(1-\delta) \tilde{Y}_{t+1}(a)+\delta Y_{t+1}^{u 2}(a)\right]
$$

- La valeur sociale d'un travailleur qui n'est pas en emploi, produit domestiquement, et est de type 2, c'est-à-dire avec des compétences professionnelles non dépréciées, issues de sa formation passée :

$$
\begin{aligned}
Y_{t}^{u 2}(a)= & b+\beta\left[p_{t} \tilde{Y}_{t+1}(a)+\left(1-p_{t}\right)(1-\pi) Y_{t+1}^{u 2}(a)\right. \\
& +\left(1-p_{t}\right) \pi\left\{\begin{array}{cc}
Y_{t+1}^{u 1}(a) & \text { si } a \geq a_{t+1}^{\star} \\
Y_{t+1}^{u 0}(a) & \text { si } a<a_{t+1}^{\star}
\end{array}\right]
\end{aligned}
$$


- Pour un travailleur de type 1 sans emploi, la valeur sociale fait intervenir le coût de formation qu'il faut payer à la période suivante en cas de réemploi:

$$
Y_{t}^{u 1}(a)=b+\beta \begin{cases}p_{t}\left(\tilde{Y}_{t+1}(a)-\gamma_{F}\right)+\left(1-p_{t}\right) Y_{t+1}^{u 1}(a) & \text { si } a \geq a_{t+1}^{\star} \\ p_{0, t} \hat{Y}_{t+1}(a)+\left(1-p_{0, t}\right) Y_{t+1}^{u 0}(a) & \text { si } a<a_{t+1}^{\star}\end{cases}
$$

- où $\hat{Y}_{t}(a)$ définit la valeur sociale d'un emploi occupé par un travailleur qui n'est pas apte à être formé (et ne le sera jamais sous l'hypothèse $a_{t}^{\star}<a_{t+1}^{\star}$ ) donnée par:

$$
\hat{Y}_{t}(a)=a+\beta\left[(1-\delta) \hat{Y}_{t+1}(a)+\delta Y_{t+1}^{u 0}(a)\right]
$$

- Pour le travailleur de type 0 sans emploi, on a finalement:

$$
Y_{t}^{u 0}(a)=b+\beta\left[p_{0, t} \hat{Y}_{t+1}(a)+\left(1-p_{0, t}\right) Y_{t+1}^{u 0}(a)\right]
$$

Le seuil d'aptitude optimal, $a_{t}^{\star} \forall t$, vérifie $\tilde{Y}_{t}\left(a_{t}^{\star}\right)-\gamma_{F}=\hat{Y}_{t}\left(a_{t}^{\star}\right)$. On est alors en mesure de montrer que: (cf. Annexe D)

$\Delta a_{T-1}^{\star}=\gamma_{f}$

$\Delta a_{T-2}^{\star}=\frac{\gamma_{f}}{\sum_{i=0}^{1} \beta^{i}(1-\delta)^{i}}$

$\Delta a_{t}^{\star}=\frac{\gamma_{f}-\left\{\sum_{i=0}^{T-3-t} \beta^{i+1} \delta(1-\delta)^{i}\left[Y_{t+1+i}^{u 2}\left(a_{t}^{\star}\right)-Y_{t++1+i}^{u 0}\left(a_{t}^{\star}\right)\right]\right\}}{\sum_{i=0}^{T-1-t} \beta^{i}(1-\delta)^{i}}, \forall t \leq T-3$

\subsection{Choix des employeurs vs. choix optimaux}

La comparaison des choix des employeurs et des décisions optimales de formation permet de généraliser les résultats de $\mathrm{BC}$ dans un cadre de cycle de vie : si, et seulement si, $\alpha=1$, c'est à dire si les salariés ont tout le pouvoir de fixation des salaires, les décisions des employeurs sont optimales. Dans le cas contraire, les employeurs ne forment pas assez, c'est à dire qu'il sont trop exigeants en termes d'aptitude minimale.

On notera en particulier que, pour $t=T-3$, il résulte:

$$
\begin{gathered}
\Delta a_{T-3}^{\star}\left[\left\{\sum_{i=0}^{2} \beta^{i}(1-\delta)^{i}\right\}+\beta^{2} \delta p_{T-2}\right]=\gamma_{f}-\left(a_{T-3}^{\star}-b\right) \beta^{2} \delta\left(p_{T-2}-p_{0, T-2}\right) \\
\Delta \tilde{a}^{T-3}\left[\left\{\sum_{i=0}^{2} \beta^{i}(1-\delta)^{i}\right\}+\alpha \beta^{2} \delta p_{T-2}\right]=\gamma_{f}-\left(\tilde{a}^{T-3}-b\right) \alpha \beta^{2} \delta\left(p_{T-2}-p_{0, T-2}\right)
\end{gathered}
$$


Dans chacune de ces expressions, trois termes essentiels interviennent:

- $\sum_{i=0}^{2} \beta^{i}(1-\delta)^{i}$ représente un effet capitalisation "classique" lié à la taille de l'horizon de rentabilisation des investissements en formation continue; ce terme intervient de façon équivalente dans les deux expressions (équilibre et optimum)

- $\beta^{2} \delta p_{T-2}$ représente le terme associé à l'externalité de débauchage, parfaitement internalisée par un planificateur; ceci représente la prise en compte de la rentabilisation du coût de formation au delà de la destruction de l'emploi qui survient avec une probabilité $\delta$ et qui pourra donner lieu à un ré-emploi des compétences transférables avec une probabilité $p_{T-2}$ (au-delà de $T-2$ l'horizon de rentabilisation est nul). Ce phénomène apparaît comme pondéré par le pouvoir de négociation des travailleurs quand il s'agit du choix de formation par les entreprises.

- L'externalité de chômage (manque à gagner en termes de production marchande vs. domestique, $a-b$ ) est conditionnée par l'écart de probabilités $\bar{p}_{T-2}-p_{0, T-2}$, qui tend à réduire le seuil exigé pour accéder à la formation et bénéficier d'une probabilité d'embauche plus élevée. Comme précédemment, cet impact est pondéré par $\alpha$ dans l'expression du seuil d'équilibre.

\section{3 Évaluations quantitatives des externalités sociales de la formation continue tout au long du cycle de vie}

Il est alors possible de procéder à une illustration quantitative des implications de ce modèle, afin d'examiner le rôle et l'incidence des externalités sociales de formation continue identifiées, selon l'âge des travailleurs.

\section{1 Étalonnage des paramètres structurels du modèle}

Afin de mettre en évidence les principales propriétés du modèle, nous proposons ici une illustration quantitative calibrée sur données françaises.

Ce modèle à l'état stationnaire est étalonné selon une périodicité trimestrielle, en considérant un âge de retraite $T=62$ ans. De manière classique, on considère $\beta=0.99, b=0.4$ et $\alpha=0.5$. Ensuite, suivant en cela Belan et Chéron [2014] nous supposons $\Delta=0.25$ et $\pi=1 / 2$ traduisant que, en situation de turbulence économique importante, les bienfaits en termes de productivité de la formation continue disparaissent en moyenne au bout de 6 mois de chômage. Concernant les paramètres caractérisant les transitions sur le marché du travail, nous nous basons sur les travaux de Hairault, Le Barbanchon et Sopraseuth [2014], de sorte que nous reproduisons une durée moyenne d'emploi égale à 28 trimestres et une durée moyenne de chômage d'environ 1 an. Ceci nous conduit à supposer $\delta=1 / 28$ et, parallèlement, à considérer $p_{t}=p=1 / 3$ et $p_{0, t}=p_{0}=0.5 p$. Nous examinerons différentes variantes s'agissant des valeurs de ses probabilités de reprise d'emploi, afin d'évaluer la robustesse des résultats quantitatifs obtenus. Enfin, la distribution des aptitudes est supposée définie sur un intervalle discret $a \in[1, \bar{a}]$ avec un étalonnage de référence supposant $\bar{a}=3$, 
Figure 2: L'évolution avec l'âge des seuils de performance individuelle requis pour être formé (étalonnage de référence)
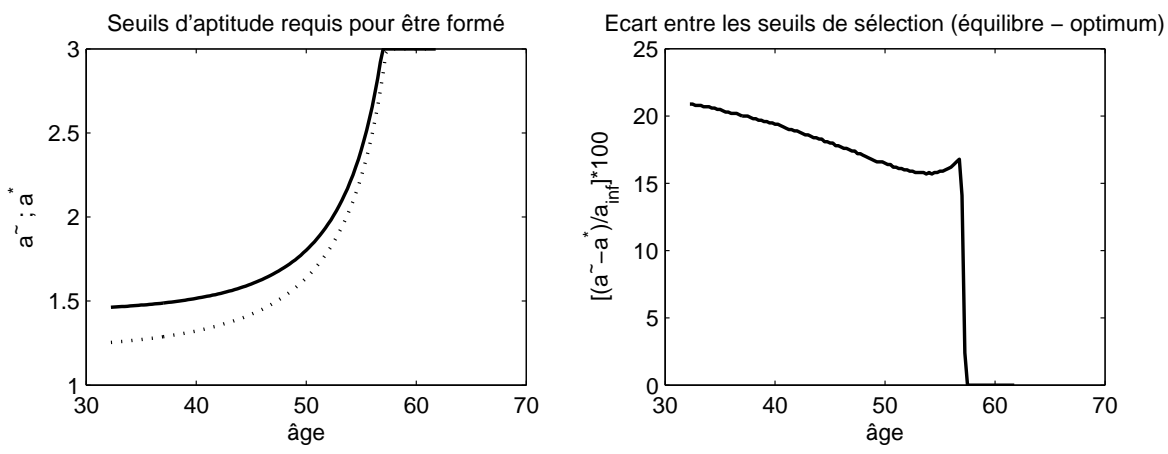

- sélection des entreprises

sélection optimale

et des coûts de formation $\gamma_{F}=12$ (équivalent à la calibration retenue dans Belan et Chéron [2014]), soit un coût de formation continue représentant entre $10 \%$ et plus de $30 \%$ de la valeur de la productivité obtenue sur la durée de l'emploi, selon le niveau de performance individuelle (indicateur défini par $\gamma_{f} /[\delta(1+\Delta) \bar{a}]$ ).

\subsection{L'évolution en fonction de l'âge de la sélection à l'entrée en formation continue, et de son écart par rapport à la sélection optimale}

La figure 2 retrace la dynamique des seuils d'aptitude requis pour accéder à la formation professionnelle, telle que l'implique notre étalonnage de référence. Nous supposons notamment ici un départ à la retraite à 62 ans, et considérons que les performances individuelles en termes de productivité (aptitudes) peuvent varier de 1 à 3 selon les travailleurs. De même, à ce stade, il n'est pas supposé que les seniors sont confrontés à un problème spécifique d'employabilité, c'est à dire confrontés à des délais de reprise d'emploi relativement plus longs s'ils ne sont pas formés.

Nous le verrons par la suite, la prise en considération de ce phénomène avéré ne fera que renforcer nos principales conclusions. Cette figure indique tout d'abord (graphique de gauche) que, selon les simulations de notre modèle, la sélection à l'entrée en formation continue augmente avec l'âge, avec une pente qui s'accentue fortement au-delà de 50 ans. Eu égard aux données présentées en introduction (Figure 1), ceci souligne évidemment la pertinence empirique de notre approche. Mais au-delà de ce diagnostic positif, il en ressort également des résultats normatifs que notre analyse structurelle permet de bien identifier. La sélection optimale à l'entrée en formation suit en effet un profil dynamique avec l'âge similaire, mais avec un écart vis-à-vis de la sélection pratiquée par les entreprises qui évolue de façon non monotone - particularité sur laquelle nous reviendrons dans un instant -, et qui chute à zéro 
au-delà d'un certain âge, ici évalué à 57 ans (graphique de droite). ${ }^{9}$

Cet écart entre la sélection pratiquée par les entreprises et la sélection optimale résulte, on l'a déjà évoqué, de la présence de deux types d'externalités, de débauchage et de chômage, faisant que le rendement social de la formation continue est supérieur à son rendement privé. Ceci est responsable d'une insuffisance d'accès à la formation professionnelle. Néanmoins, nous notons donc ici que la prise en considération de la sortie du marché du travail vers la retraite est à l'origine d'une convergence du rendement social de la formation vers son rendement privé en fin de carrière, l'écart entre les deux rendements devenant même nul au-delà d'un certain âge. Les externalités deviennent en effet trop faibles à l'approche de la retraite, de sorte qu'il n'est plus rentable d'engager des dépenses de formation professionnelle pour les travailleurs de plus de 57 ans, y compris sur la base du rendement social de ces dépenses. ${ }^{10}$

\subsection{L'impact relatif de l'externalité de chômage et la question de l'employabilité des seniors}

Il est important de souligner une seconde propriété mise en évidence par cette évaluation quantitative; elle renvoie à l'évolution non monotone avec l'âge de l'écart entre la sélection effectuée par les entreprises et la sélection optimale à l'entrée en formation continue. Ce phénomène, qui intervient modestement sur la figure 2 entre 50 et 57 ans (graphique de droite), se trouve renforcé s'il est a fortiori tenu compte du problème spécifique d'employabilité rencontré par les seniors.

La figure 3 considère ainsi deux variantes de notre étalonnage de référence: (i) sur le graphique de gauche, on suppose que la formation n'a pas d'incidence sur l'employabilité ultérieure du travailleur, c'est à dire que les probabilités de reprise d'emploi sont ici supposées indépendantes du capital humain (noté $p=p_{0}$ ), et (ii) à l'inverse sur le graphique de droite, il est considéré que les seniors, soit de plus de 55 ans, soit de plus de 50 ans, ont une probabilité nulle de reprise d'emploi quand ils n'ont pas un capital humain "à jour", ce qui est noté respectivement $p_{0}(55-62)=0$ et $p_{0}(50-62)=0$. L'intérêt d'examiner ces différentes variantes est qu'elles permettent d'évaluer le rôle de l'externalité de chômage, qui se trouve renforcée quand on considère la perte d'employabilité des seniors non formés, et à l'inverse évincée s'il est supposé que le capital humain n'a pas d'impact sur les probabilités de reprise d'emploi.

Sur le graphique de gauche de la figure 3, on notera donc tout d'abord que c'est bien l'existence d'une externalité de chômage (absente a contrario si $p=p_{0}$ ) qui est respons-

\footnotetext{
${ }^{9} \mathrm{Si}$ on considère une distribution continue sur un support infini des aptitudes, des propriétés qualitativement similaires peuvent être obtenues, avec un écart de sélection entre les choix des entreprises et les choix optimaux qui tend vers zéro, sans jamais l'atteindre, contrairement à ce que nous obtenons ici puisque nous avons considéré une distribution discrète et bornée des aptitudes individuelles.

${ }^{10}$ Par ailleurs, dans la lignée des résultats obtenus par Belan et Chéron [2014] on peut également noter que l'écart de sélection entre équilibre et optimum augmente avec la turbulence (propriété non reportée ici): l'impact de la turbulence sur l'externalité de chômage domine celui sur l'externalité de débauchage, suggérant qu'il est globalement souhaitable de renforcer le soutien à la formation continue lorsque le phénomène de dépréciation du capital humain général au chômage s'accélère.
} 
Figure 3: Seuils minimum requis pour être formé et le rôle de l'externalité de chômage

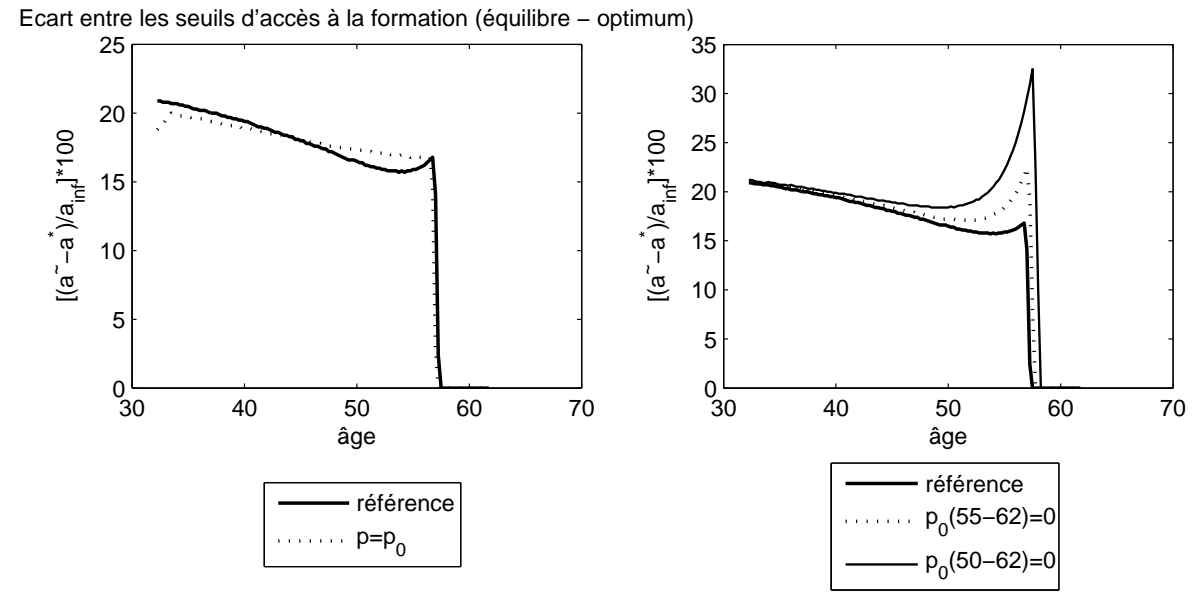

able de la dynamique non monotone de l'écart de sélection à l'entrée en formation: l'effet d'horizon court, qui tend à accroître cette sélection à mesure que la retraite approche, apparaît en effet décalé à l'optimum, du fait de l'externalité de chômage. En d'autres termes, l'élévation de la sélection à l'entrée en formation pratiquée par les entreprises intervient trop précocement et trop abruptement au regard de ce que suggère de faire la prise en compte du rendement social de la formation. Évidemment, si les seniors sont a fortiori concernés par une perte d'employabilité faisant que, s'il ne bénéficient plus de formation, leur probabilité de reprise d'emploi tend vers zéro, on comprend que le poids de l'externalité de chômage augmente. C'est ce qui apparaît sur le graphique de droite de la figure 3. On peut notamment observer que pour les travailleurs de 53-57 ans l'excès de sélection à l'entrée en formation continue devient plus important que pour les plus jeunes. Ceci plaide donc très clairement pour la mise en oeuvre d'une politique ciblée en faveur de cette tranche d'âge, alors qu'au delà de 57 ans, il ne semble pas souhaitable de chercher à réguler, ou contrecarrer la pratique des entreprises qui ne proposent pas de formation à ces travailleurs.

\subsection{Analyse de sensibilité des résultats quantitatifs}

Évidemment, on peut s'interroger sur la "robustesse" de l'âge seuil, 57 ans, autour duquel nos simulations indiquent qu'il faut différencier fortement la politique de soutien à la formation continue : pas d'incitation au-delà de 57 ans et, à l'inverse, une politique très "pro-active" juste en dessous, c'est-à-dire pour les 50-57 ans.

Au préalable, il est important de noter que, si cette différentiation autour d'un âge "critique" constitue un résultat robuste, celui-ci est, par construction, dépendant de l'âge de départ à la retraite, supposé égal à 62 ans dans nos simulations (en ligne avec l'âge minimum légal). L'ensemble des comportements identifiés ne se définissent pas en effet en fonction de l'âge biologique, mais bien de la distance à la sortie du marché du travail. Ceci étant dit, on peut considérer différentes variantes de notre étalonnage, afin d'évaluer la sensibilité de 
l'âge autour duquel doit se faire cette différenciation en matière de soutien à la formation professionnelle continue.

Ces variantes concernent notamment l'amplitude de l'hétérogénéité des individus en termes de performance productive, de 1 à 3 dans notre étalonnage de référence, vs. 1 à 2 ou 1 à 4 dans nos variantes. On montre ainsi que l'âge seuil au-delà duquel il n'est pas nécessaire d'inciter les employeurs à dépenser pour la formation continue de leurs salariés va de 55 ans dans le cas d'une distribution plus concentrée, à un peu plus de 58 ans pour la configuration où une hétérogénéité plus grande est considérée (figure 4). Finalement, nous proposons également d'examiner la sensibilité de ce résultat à la taille du coût de formation supposée (voir l'annexe pour une discussion de l'étalonnage de référence). Les simulations indiquent que cette sensibilité reste modeste puisque, en réduisant de $50 \%$ le coût de formation, l'âge seuil mesuré est simplement décalé de 57 à 58 ans (figure 5).

Figure 4: Seuils minimum requis pour être formé : test de sensibilité à l'hétérogénéité des travailleurs

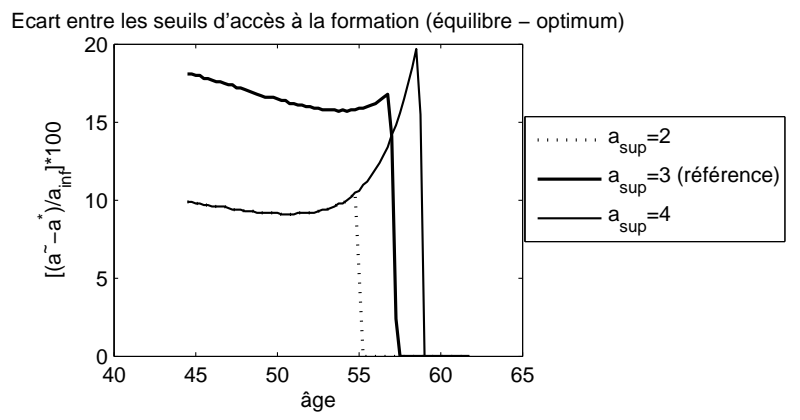

Figure 5: Seuils minimum requis pour être formé : test de sensibilité au coût de formation

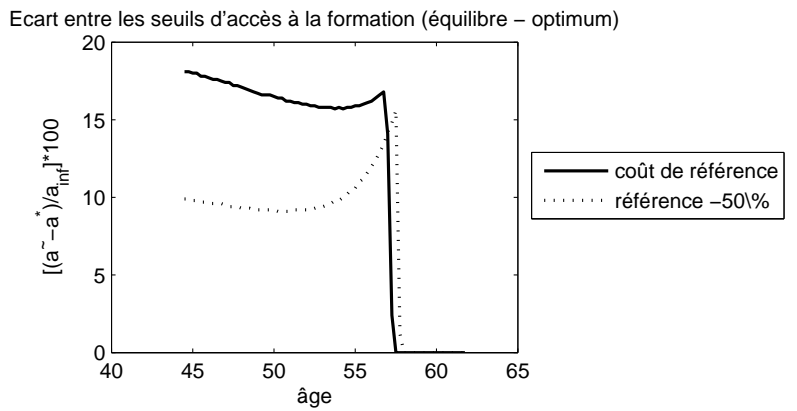




\section{Conclusion}

Dans cette étude, nous présentons un cadre théorique permettant d'analyser les interactions entre investissement en capital humain, turbulence économique et cycle de vie afin d'examiner la dynamique des externalités sociales liées à la formation générale: D'une part, un travailleur est susceptible de mobiliser les compétences acquises au cours d'une formation générale dans des emplois ultérieurs, c'est l'externalité de débauchage mise en évidence par Acemoglu et Pischke [1999]. D'autre part, Belan et Chéron [2011, 2014] ont souligné que, si les individus ne bénéficiant pas de formation professionnelle sont confrontés à des durées de chômage relativement plus longues, le refus par les employeurs de former certains travailleurs est à l'origine d'une externalité de chômage.

L'existence de telles externalités conduit à exclure certains travailleurs du système de formation professionnelle, à tort du point de vue de ce qui serait socialement souhaitable. Il convient donc de proposer une politique de soutien à la formation ciblée vers certains types de travailleurs. La principale contribution de ce papier est en effet de positionner cette problématique des externalités sociales de la formation continue dans la perspective du cycle de vie du travailleur, et de souligner qu'il serait nécessaire de mettre en place une politique d'incitation à la formation dépendante de l'âge. Nous montrons en effet que les employeurs tendent à accroître trop tôt dans le cycle de vie des travailleurs leur critère d'accès à la formation continue relativement à ce que suggérerait de faire la prise en compte des externalités sociales. En revanche, en toute fin de carrière (à l'approche de la retraite) le rendement social converge vers le rendement privé de la formation et tend vers zéro. Au bilan, ceci plaide donc pour une politique incitative non monotone avec l'âge, croissante jusqu'à un certain âge, puis décroissante ensuite.

Sur le design précis et chiffré des politiques incitatives à mettre en oeuvre, il serait bien entendu nécessaire d'enrichir l'analyse proposée, notamment en explicitant les probabilités de transition du chômage vers l'emploi, au travers d'un processus d'appariement. Il est toutefois d'ores et déjà possible de caractériser la nature des politiques susceptibles de faire coïncider équilibre et optimum dans ce contexte. Il s'agit simplement d'une déclinaison au cours du cycle de vie du principe de subventions déjà proposé par Belan et Chéron [2011,2014] . Concrètement, des subventions à la formation continue financées par des prélèvements forfaitaires, et un taux modulé en fonction de l'âge, constituent un outil de politique publique en mesure de rendre optimal les choix de formation tout au long de la carrière des travailleurs. ${ }^{11}$

\footnotetext{
${ }^{11}$ Le coût net de la formation pour l'employeur peut dans ce cadre être noté $\gamma_{f}\left(1-s_{t}\right)$ où $s_{t}$ est le taux de subvention dépendant de l'âge
} 


\section{Références}

Acemoglu (1997) "Training and innovation in an imperfect labor market", Review of Economic Studies, Wiley Blackwell, vol. 64, pages 445-464

Acemoglu et Pischke (1998), "Why do firms train? Theory and evidence", The Quaterly Journal of Economics, MIT Press, vol. 113, pages 78-118

Acemoglu et Pischke (1999a), "Beyond Becker : Training in imperfect Labor markets", Economic Journal, Royal Economic Society, vol. 109(453), pages F112-42

Acemoglu et Pischke (1999b), "The structure of wages and investment in general training", Journal of Political Economy, University of Chicago Press, vol. 107, pages 539-572

Becker (1964), "Human capital. A theoretical an empirical analysis, with special reference to education", University of Chicago Press

Belan et Chéron (2011), "Chômage d'équilibre, dépréciation du capital humain et subvention optimale à la formation", Revue d'Economie Politique, vol. Mars-Avril

Belan et Chéron (2014), "Turbulence, training and unemployment", Labour Economics, vol. 27, pages $16-29$

Chéron (2007), "Faut-il subventionner la formation professionnelle des seniors?", EDHEC position paper

Chéron (2012), "De la modulation des subventions à la formation continue", Edhec Position Paper

Chéron, Hairault, Langot (2011), "Age-Dependent Employment Protection", Economic Journal, Royal Economic Society, vol. 121(557), pages 1477-1504

Chéron, Hairault et Langot (2013), "Life-cycle equilibrium unemployment", Journal of Labor Economics, University of Chicago Press, vol. 31, pages 843-882

Hairault, Le Barbanchon, Sopraseuth (2014), "The cyclicality of the separation and job finding rates in France", IZA Discussion Paper 6906

Ljungqvist et Sargent (1998), "The european unemployment dilemma", Journal of Political Economy, University of Chicago Press, vol. 106, pages 514-550

Ljungqvist et Sargent (2004), "European unemployment and turbulence revisited in a matching model", Journal of the European Economic Association, MIT Press, vol. 2, pages 456-468 
Ljungqvist et Sargent (2007), "Understanding European unemployment with matching and search-island models", Journal of Monetary Economics, Elsevier, vol. 54, pages 2139-2179 


\section{A Les flux sur le marché du travail}

Soient $u_{j}^{t}(a)$ et $e_{j}^{t}(a)$ respectivement le chômage et l'emploi des individus de type $j$ et d'âge $t$ ayant un niveau d'aptitude $a$.

En $t=0$ :

A cet âge, tous les individus entrent sur le marché du travail avec le statut de chômeur et des compétences à jour, soit :

- $\forall a$ :

$$
\begin{aligned}
& u_{2}^{0}(a)=f(a) \\
& e_{2}^{0}(a)=0
\end{aligned}
$$

En $t=1$ :

A partir de cette période, on détermine à chaque âge un seuil d'aptitude critique $\tilde{a}^{t}$ représentant l'aptitude requise pour être formé. On note que certains chômeurs de type 2 peuvent avoir subi une dépréciation de leur capital humain au cours de leur épisode de chômage (avec une probabilité $\pi$ ) : Ils deviennent dans ce cas chômeurs de type 0 si leur aptitude se situe sous le seuil $\tilde{a}^{1}$ ou chômeurs de type 1 si leur aptitude est supérieure ou égale à $\tilde{a}^{1}$.

- $\forall a<\tilde{a}^{1}$,

$u_{0}^{1}(a)=f(a)\left[1-p_{0}^{0}(a)\right] \pi$

$e_{0}^{1}(a)=0$

$u_{2}^{1}(a)=f(a)\left[1-p^{0}(a)\right](1-\pi)$

$e_{2}^{1}(a)=f(a) p^{0}(a)$

- $\forall \geq \tilde{a}^{1}$,

$$
\begin{aligned}
& u_{1}^{1}(a)=f(a)\left[1-p^{0}(a)\right] \pi \\
& e_{1}^{1}(a)=0 \\
& u_{2}^{1}(a)=f(a)\left[1-p^{0}(a)\right](1-\pi) \\
& e_{2}^{1}(a)=f(a) p^{0}(a)
\end{aligned}
$$


En $t=2$ :

A partir de cette période, on note que certains chômeurs éligibles à une formation professionnelle à la période précédente peuvent désormais avoir un niveau d'aptitude trop faible pour être formés et devenir chômeurs de type 0 .

- $\forall a<\tilde{a}^{1}$ :

$$
\begin{aligned}
& u_{0}^{2}(a)=u_{0}^{1}(a)\left[1-p_{0}^{1}(a)\right]+u_{2}^{1}(a)\left[1-p^{1}(a)\right] \pi \\
& e_{0}^{2}(a)=u_{0}^{1}(a) p_{0}^{1}(a) \\
& u_{2}^{2}(a)=u_{2}^{1}(a)\left[1-p^{1}(a)\right](1-\pi)+\delta e_{2}^{1}(a) \\
& e_{2}^{2}(a)=(1-\delta) e_{2}^{1}(a)+u_{2}^{1}(a) p^{1}(a)
\end{aligned}
$$

- $\forall a \in\left[\tilde{a}^{1} ; \tilde{a}^{2}[:\right.$

$$
\begin{aligned}
& u_{0}^{2}(a)=u_{1}^{1}(a)\left[1-p_{0}^{1}(a)\right]+u_{2}^{1}(a)\left[1-p^{1}(a)\right] \pi \\
& e_{0}^{2}(a)=u_{1}^{1}(a) p_{0}^{1}(a) \\
& u_{2}^{2}(a)=u_{2}^{1}(a)\left[1-p^{1}(a)\right](1-\pi)+\delta e_{2}^{1}(a) \\
& e_{2}^{2}(a)=(1-\delta) e_{2}^{1}(a)+u_{2}^{1}(a) p^{1}(a)
\end{aligned}
$$

- $\forall a \geq \tilde{a}^{2}$ :

$$
\begin{aligned}
& u_{1}^{2}(a)=u_{1}^{1}(a)\left[1-p^{1}(a)\right]+u_{2}^{1}(a)\left[1-p^{1}(a)\right] \pi \\
& e_{1}^{2}(a)=u_{1}^{1}(a) p^{1}(a) \\
& u_{2}^{2}(a)=u_{2}^{1}(a)\left[1-p^{1}(a)\right](1-\pi)+\delta e_{2}^{1}(a) \\
& e_{2}^{2}(a)=(1-\delta) e_{2}^{1}(a)+u_{2}^{1}(a) p^{1}(a)
\end{aligned}
$$


$\forall t \in[3 ; T-1]:$

A partir de $t=3$, on obtient une structure des flux qui se reproduit jusqu'à l'âge de la retraite.

- $\forall a<\tilde{a}^{t-1}$,

$$
\begin{aligned}
& u_{0}^{t}(a)=u_{0}^{t-1}(a)\left[1-p_{0}^{t-1}(a)\right]+u_{2}^{t-1}(a)\left[1-p^{t-1}(a)\right] \pi+\delta e_{0}^{t-1}(a) \\
& e_{0}^{t}(a)=(1-\delta) e_{0}^{t-1}(a)+u_{0}^{t-1}(a) p_{0}^{t-1}(a) \\
& u_{2}^{t}(a)=u_{2}^{t-1}(a)\left[1-p^{t-1}(a)\right](1-\pi)+\delta e_{2}^{t-1}(a) \\
& e_{2}^{t}(a)=(1-\delta) e_{2}^{t-1}(a)+u_{2}^{t-1}(a) p^{t-1}(a)
\end{aligned}
$$

- $\forall a \in\left[\tilde{a}^{t-1} ; \tilde{a}^{t}[\right.$,

$$
\begin{aligned}
& u_{0}^{t}(a)=u_{1}^{t-1}(a)\left[1-p_{0}^{t-1}(a)\right]+u_{2}^{t-1}(a)\left[1-p^{t-1}(a)\right] \pi \\
& e_{0}^{t}(a)=u_{1}^{t-1}(a) p_{0}^{t-1}(a) \\
& u_{2}^{t}(a)=u_{2}^{t-1}(a)\left[1-p^{t-1}(a)\right](1-\pi)+\delta\left[e_{1}^{t-1}(a)+e_{2}^{t-1}(a)\right] \\
& e_{2}^{t}(a)=(1-\delta)\left[e_{1}^{t-1}(a)+e_{2}^{t-1}(a)\right]+u_{2}^{t-1}(a) p^{t-1}(a)
\end{aligned}
$$

- $\forall a \geq \tilde{a}^{t}$

$$
\begin{aligned}
& u_{1}^{t}(a)=u_{1}^{t-1}(a)\left[1-p^{t-1}(a)\right]+u_{2}^{t-1}(a)\left[1-p^{t-1}(a)\right] \pi \\
& e_{1}^{t}(a)=u_{1}^{t-1}(a) p^{t-1}(a) \\
& u_{2}^{t}(a)=u_{2}^{t-1}(a)\left[1-p^{t-1}(a)\right](1-\pi)+\delta\left[e_{1}^{t-1}(a)+e_{2}^{t-1}(a)\right] \\
& e_{2}^{t}(a)=(1-\delta)\left[e_{1}^{t-1}(a)+e_{2}^{t-1}(a)\right]+u_{2}^{t-1}(a) p^{t-1}(a)
\end{aligned}
$$

\section{B Les équations de salaires négociés}

Quelque soit l'âge considéré, le salaire est déterminé par une règle de partage vérifiant le critère de Nash, et est renégocié à chaque période. Soit $\alpha$ le pouvoir de négociation des travailleurs, la règle s'écrit alors de la manière suivante selon le type de travailleur considéré:

$$
\begin{aligned}
(1-\alpha)\left[E_{0}^{t}(a)-U_{0}^{t}(a)\right] & =\alpha J_{0}^{t}(a) \\
(1-\alpha)\left[E_{1}^{t}(a)-U_{1}^{t}(a)\right] & =\alpha\left[J_{1}^{t}(a)-\gamma_{F}\right] \\
(1-\alpha)\left[E_{2}^{t}(a)-U_{2}^{t}(a)\right] & =\alpha J_{2}^{t}(a)
\end{aligned}
$$


B.1 Individus de type 0 , non éligibles à la formation $\left(a<\tilde{a}^{t}\right)$

Du côté des travailleurs :

$\left[E_{0}^{t}(a)-U_{0}^{t}(a)\right]=w_{0}^{t}(a)-b+\beta(1-\delta)\left[E_{0}^{t+1}(a)-U_{0}^{t+1}(a)\right]-\beta p_{0}^{t}(a)\left[E_{0}^{t+1}(a)-U_{0}^{t+1}(a)\right]$

Du côté des firmes:

$J_{0}^{t}(a)=a-w_{0}^{t}(a)+\beta(1-\delta) J_{0}^{t+1}(a)$

D'après la règle de partage :

$(1-\alpha)\left[w_{0}^{t}(a)-b+\beta(1-\delta)\left[E_{0}^{t+1}(a)-U_{0}^{t+1}(a)\right]-\beta p_{0}^{t}(a)\left[E_{0}^{t+1}(a)-U_{0}^{t+1}(a)\right]\right]$

$=\alpha\left[a-w_{0}^{t}(a)+\beta(1-\delta) J_{0}^{t+1}(a)\right]$

On en déduit le salaire des individus de type 0 :

$$
w_{0}^{t}(a)=\alpha a+(1-\alpha) b+\alpha \beta p_{0}^{t}(a) J_{0}^{t+1}(a)
$$

Et la valeur d'un poste occupé par un individu de type 0 :

$$
J_{0}^{t}(a)=(1-\alpha)(a-b)+\beta(1-\delta) J_{0}^{t+1}(a)-\alpha \beta p_{0}^{t}(a) J_{0}^{t+1}(a)
$$

\section{B.2 Individus de type 1, éligibles à la formation pour la dernière fois $\left(\tilde{a}^{t}<a<\tilde{a}^{t+1}\right)$}

Du côté des travailleurs :

$$
\begin{aligned}
{\left[E_{1}^{t}(a)-U_{1}^{t}(a)\right]=} & w_{1}^{t}(a)-b+\beta(1-\delta)\left[E_{2}^{t+1}(a)-U_{2}^{t+1}(a)\right]-\beta p_{0}^{t}(a)\left[E_{0}^{t+1}(a)-U_{0}^{t+1}(a)\right] \\
& +\beta\left[U_{2}^{t+1}(a)-U_{0}^{t+1}(a)\right]
\end{aligned}
$$

Du côté des firmes:

$J_{1}^{t}(a)=(1+\Delta) a-w_{1}^{t}(a)+\beta(1-\delta) J_{2}^{t+1}(a)$

D'après la règle de partage :

$$
\begin{aligned}
& (1-\alpha)\left[w_{1}^{t}(a)-b+\beta(1-\delta)\left[E_{2}^{t+1}(a)-U_{2}^{t+1}(a)\right]-\beta p_{0}^{t}(a)\left[E_{0}^{t+1}(a)-U_{0}^{t+1}(a)\right]\right. \\
& \left.+\beta\left[U_{2}^{t+1}(a)-U_{0}^{t+1}(a)\right]\right]=\alpha\left[a-w_{0}^{t}(a)+\beta(1-\delta) J_{0}^{t+1}(a)\right]
\end{aligned}
$$


On en déduit le salaire des individus de type 1 :

$w_{1}^{t}(a)=\alpha\left[(1+\Delta) a-\gamma_{f}\right]+(1-\alpha) b+\alpha \beta p_{0}^{t}(a) J_{0}^{t+1}(a)-\beta(1-\alpha)\left[U_{2}^{t+1}(a)-U_{0}^{t+1}(a)\right]$

Et la valeur d'un poste occupé par un individu de type 1 :

$$
\begin{aligned}
J_{1}^{t}(a)= & (1-\alpha)[(1+\Delta) a-b]+\beta(1-\delta) J_{2}^{t+1}(a)-\alpha \beta p_{0}^{t}(a) J_{0}^{t+1}(a) \\
& +\alpha \gamma_{f}+\beta(1-\alpha)\left[U_{2}^{t+1}(a)-U_{0}^{t+1}(a)\right]
\end{aligned}
$$

\section{B.3 Individus de type 2, ayant des connaissances à jour}

Du côté des travailleurs :

$$
\begin{aligned}
& {\left[E_{2}^{t}(a)-U_{2}^{t}(a)\right]=w_{2}^{t}(a)-b+\beta(1-\delta)\left[E_{2}^{t+1}(a)-U_{2}^{t+1}(a)\right]-\beta p^{t}(a)\left[E_{2}^{t+1}(a)-U_{2}^{t+1}(a)\right]} \\
& +\beta \pi\left[1-p^{t}(a)\right]\left[U_{2}^{t+1}(a)-U_{0}^{t+1}(a)\right]
\end{aligned}
$$

Du côté des firmes:

$J_{2}^{t}(a)=(1+\Delta) a-w_{2}^{t}(a)+\beta(1-\delta) J_{2}^{t+1}(a)$

D'après la règle de partage :

$$
\begin{aligned}
& (1-\alpha)\left[w_{2}^{t}(a)-b+\beta(1-\delta)\left[E_{2}^{t+1}(a)-U_{2}^{t+1}(a)\right]-\beta p^{t}(a)\left[E_{2}^{t+1}(a)-U_{2}^{t+1}(a)\right]\right. \\
& \left.+\beta \pi\left[1-p^{t}(a)\right]\left[U_{2}^{t+1}(a)-U_{0}^{t+1}(a)\right]\right]=\alpha\left[(1+\Delta) a-w_{2}^{t}(a)+\beta(1-\delta) J_{2}^{t+1}(a)\right]
\end{aligned}
$$

On en déduit le salaire des individus de type 2 :

$w_{2}^{t}(a)=\alpha(1+\Delta) a+(1-\alpha) b+\alpha \beta p^{t}(a) J_{2}^{t+1}(a)-\beta \pi(1-\alpha)\left[1-p^{t}(a)\right]\left[U_{2}^{t+1}(a)-U_{0}^{t+1}(a)\right]$

Et la valeur d'un poste occupé par un individu de type 0 :

$$
\begin{aligned}
J_{2}^{t}(a)= & (1-\alpha)[(1+\Delta) a-b]+\beta(1-\delta) J_{2}^{t+1}(a)-\alpha \beta p^{t}(a) J_{2}^{t+1}(a) \\
& +\beta \pi(1-\alpha)\left[1-p^{t}(a)\right]\left[U_{2}^{t+1}(a)-U_{0}^{t+1}(a)\right]
\end{aligned}
$$




\section{Seuils de formation à l'équilibre}

La politique de formation des entreprises consiste à choisir $\tilde{a}^{t} \forall t$ tel que $J_{1}^{t}\left(\tilde{a}^{t}\right)-\gamma_{f}=J_{0}^{t}\left(\tilde{a}^{t}\right)$.

On pose alors :

$$
\begin{aligned}
& (1-\alpha)\left[(1+\Delta) a-b-\gamma_{f}\right]+\beta(1-\delta) J_{2}^{t+1}(a)-\alpha \beta p_{0}^{t}(a) J_{0}^{t+1}(a) \\
& +\beta(1-\alpha)\left[U_{2}^{t+1}(a)-U_{0}^{t+1}(a)\right]=(1-\alpha)(a-b)+\beta(1-\delta) J_{0}^{t+1}(a)-\alpha \beta p_{0}^{t}(a) J_{0}^{t+1}(a)
\end{aligned}
$$

On obtient alors la condition suivante :

$$
\Delta \tilde{a}^{t}=\gamma_{f}-\frac{\beta(1-\delta)}{1-\alpha}\left[J_{2}^{t+1}\left(\tilde{a}^{t}\right)-J_{0}^{t+1}\left(\tilde{a}^{t}\right)\right]-\beta\left[U_{2}^{t+1}\left(\tilde{a}^{t}\right)-U_{0}^{t+1}\left(\tilde{a}^{t}\right)\right]
$$

En utilisant les fonctions-valeurs et en remplaçant les salaires négociés par leurs expressions, on montre que :

$$
\begin{aligned}
J_{2}^{t+1}\left(\tilde{a}^{t}\right)-J_{0}^{t+1}\left(\tilde{a}^{t}\right)= & (1-\alpha) \Delta \tilde{a}^{t}+\beta(1-\delta)\left[J_{2}^{t+2}\left(\tilde{a}^{t}\right)-J_{0}^{t+2}\left(\tilde{a}^{t}\right)\right] \\
& -\alpha \beta\left[p^{t+1}(a) J_{2}^{t+2}\left(\tilde{a}^{t}\right)-p_{0}^{t+1}(a) J_{0}^{t+2}\left(\tilde{a}^{t}\right)\right] \\
& +\beta \pi(1-\alpha)\left[1-p^{t+1}(a)\right]\left[U_{2}^{t+2}\left(\tilde{a}^{t}\right)-U_{0}^{t+2}\left(\tilde{a}^{t}\right)\right] \\
U_{2}^{t+1}\left(\tilde{a}^{t}\right)-U_{0}^{t+1}\left(\tilde{a}^{t}\right)= & \frac{\alpha \beta}{1-\alpha}\left[p^{t+1}(a) J_{2}^{t+2}\left(\tilde{a}^{t}\right)-p_{0}^{t+1}(a) J_{0}^{t+2}\left(\tilde{a}^{t}\right)\right] \\
& +\beta\left[U_{2}^{t+2}\left(\tilde{a}^{t}\right)-U_{0}^{t+2}\left(\tilde{a}^{t}\right)\right] \\
& -\beta \pi\left[1-p^{t+1}(a)\right]\left[U_{2}^{t+2}\left(\tilde{a}^{t}\right)-U_{0}^{t+2}\left(\tilde{a}^{t}\right)\right]
\end{aligned}
$$

On remarque alors que :

$$
\begin{aligned}
J_{2}^{t+1}\left(\tilde{a}^{t}\right)-J_{0}^{t+1}\left(\tilde{a}^{t}\right)= & (1-\alpha) \Delta \tilde{a}^{t}+\beta(1-\delta)\left[J_{2}^{t+2}\left(\tilde{a}^{t}\right)-J_{0}^{t+2}\left(\tilde{a}^{t}\right)\right] \\
& -(1-\alpha)\left[U_{2}^{t+1}\left(\tilde{a}^{t}\right)-U_{0}^{t+1}\left(\tilde{a}^{t}\right)\right] \\
& +\beta(1-\alpha)\left[U_{2}^{t+2}\left(\tilde{a}^{t}\right)-U_{0}^{t+2}\left(\tilde{a}^{t}\right)\right]
\end{aligned}
$$


On peut alors réécrire la condition initiale :

$$
\begin{aligned}
& \Delta \tilde{a}^{t}=\gamma_{f}-\beta\left[U_{2}^{t+1}\left(\tilde{a}^{t}\right)-U_{0}^{t+1}\left(\tilde{a}^{t}\right)\right]-\frac{\beta(1-\delta)}{1-\alpha}\left[(1-\alpha) \Delta \tilde{a}^{t}+\beta(1-\delta)\left[J_{2}^{t+2}\left(\tilde{a}^{t}\right)-J_{0}^{t+2}\left(\tilde{a}^{t}\right)\right]\right. \\
& \left.-(1-\alpha)\left[U_{2}^{t+1}\left(\tilde{a}^{t}\right)-U_{0}^{t+1}\left(\tilde{a}^{t}\right)\right]+\beta(1-\alpha)\left[U_{2}^{t+2}\left(\tilde{a}^{t}\right)+U_{0}^{t+2}\left(\tilde{a}^{t}\right)\right]\right] \\
& =\gamma_{f}-\Delta \tilde{a}^{t}[\beta(1-\delta)]-\frac{\beta^{2}(1-\delta)^{2}}{1-\alpha}\left[J_{2}^{t+2}\left(\tilde{a}^{t}\right)-J_{0}^{t+2}\left(\tilde{a}^{t}\right)\right]-\beta \delta\left[U_{2}^{t+1}\left(\tilde{a}^{t}\right)-U_{0}^{t+1}\left(\tilde{a}^{t}\right)\right] \\
& -\beta^{2}(1-\delta)\left[U_{2}^{t+2}\left(\tilde{a}^{t}\right)-U_{0}^{t+2}\left(\tilde{a}^{t}\right)\right] \\
& =\gamma_{f}-\Delta \tilde{a}^{t}[\beta(1-\delta)]-\frac{\beta^{2}(1-\delta)^{2}}{1-\alpha}\left[(1-\alpha) \Delta \tilde{a}^{t}+\beta(1-\delta)\left[J_{2}^{t+3}\left(\tilde{a}^{t}\right)-J_{0}^{t+3}\left(\tilde{a}^{t}\right)\right]\right. \\
& \left.-(1-\alpha)\left[U_{2}^{t+2}\left(\tilde{a}^{t}\right)-U_{0}^{t+2}\left(\tilde{a}^{t}\right)\right]+\beta(1-\alpha)\left[U_{2}^{t+3}\left(\tilde{a}^{t}\right)+U_{0}^{t+3}\left(\tilde{a}^{t}\right)\right]\right] \\
& -\beta \delta\left[U_{2}^{t+1}\left(\tilde{a}^{t}\right)-U_{0}^{t+1}\left(\tilde{a}^{t}\right)\right]-\beta^{2}(1-\delta)\left[U_{2}^{t+2}\left(\tilde{a}^{t}\right)-U_{0}^{t+2}\left(\tilde{a}^{t}\right)\right] \\
& =\gamma_{f}-\Delta \tilde{a}^{t}\left\{\sum_{i=1}^{2} \beta^{i}(1-\delta)^{i}\right\}-\frac{\beta^{3}(1-\delta)^{3}}{1-\alpha}\left[J_{2}^{t+3}\left(\tilde{a}^{t}\right)-J_{0}^{t+3}\left(\tilde{a}^{t}\right)\right] \\
& -\beta \delta\left[U_{2}^{t+1}\left(\tilde{a}^{t}\right)-U_{0}^{t+1}\left(\tilde{a}^{t}\right)\right]-\beta^{2} \delta(1-\delta)\left[U_{2}^{t+2}\left(\tilde{a}^{t}\right)-U_{0}^{t+2}\left(\tilde{a}^{t}\right)\right] \\
& -\beta^{3}(1-\delta)\left[U_{2}^{t+3}\left(\tilde{a}^{t}\right)-U_{0}^{t+3}\left(\tilde{a}^{t}\right)\right] \\
& =\gamma_{f}-\Delta \tilde{a}^{t}\left\{\sum_{i=1}^{2} \beta^{i}(1-\delta)^{i}\right\}-\frac{\beta^{3}(1-\delta)^{3}}{1-\alpha}\left[(1-\alpha) \Delta \tilde{a}^{t}+\beta(1-\delta)\left[J_{2}^{t+4}\left(\tilde{a}^{t}\right)-J_{0}^{t+4}\left(\tilde{a}^{t}\right)\right]\right. \\
& \left.-(1-\alpha)\left[U_{2}^{t+3}\left(\tilde{a}^{t}\right)-U_{0}^{t+3}\left(\tilde{a}^{t}\right)\right]+\beta(1-\alpha)\left[U_{2}^{t+4}\left(\tilde{a}^{t}\right)+U_{0}^{t+4}\left(\tilde{a}^{t}\right)\right]\right] \\
& -\beta \delta\left[U_{2}^{t+1}\left(\tilde{a}^{t}\right)-U_{0}^{t+1}\left(\tilde{a}^{t}\right)\right]-\beta^{2} \delta(1-\delta)\left[U_{2}^{t+2}\left(\tilde{a}^{t}\right)-U_{0}^{t+2}\left(\tilde{a}^{t}\right)\right] \\
& -\beta^{3}(1-\delta)\left[U_{2}^{t+3}\left(\tilde{a}^{t}\right)-U_{0}^{t+3}\left(\tilde{a}^{t}\right)\right] \\
& =\gamma_{f}-\Delta \tilde{a}^{t}\left\{\sum_{i=1}^{3} \beta^{i}(1-\delta)^{i}\right\}-\frac{\beta^{4}(1-\delta)^{4}}{1-\alpha}\left[J_{2}^{t+4}\left(\tilde{a}^{t}\right)-J_{0}^{t+4}\left(\tilde{a}^{t}\right)\right] \\
& -\beta \delta\left[U_{2}^{t+1}\left(\tilde{a}^{t}\right)-U_{0}^{t+1}\left(\tilde{a}^{t}\right)\right]-\beta^{2} \delta(1-\delta)\left[U_{2}^{t+2}\left(\tilde{a}^{t}\right)-U_{0}^{t+2}\left(\tilde{a}^{t}\right)\right] \\
& -\beta^{3} \delta(1-\delta)^{2}\left[U_{2}^{t+3}\left(\tilde{a}^{t}\right)-U_{0}^{t+3}\left(\tilde{a}^{t}\right)\right]-\beta^{3}(1-\delta)\left[U_{2}^{t+3}\left(\tilde{a}^{t}\right)-U_{0}^{t+3}\left(\tilde{a}^{t}\right)\right]
\end{aligned}
$$


Par itération, on montre que :

$$
\Delta \tilde{a}^{t}=\frac{\gamma_{f}-\left\{\sum_{i=0}^{T-3-t} \beta \delta[\beta(1-\delta)]^{i}\left[U_{2}^{t+1+i}\left(\tilde{a}^{t}\right)-U_{0}^{t+1+i}\left(\tilde{a}^{t}\right)\right]\right\}}{\sum_{i=0}^{T-1-t}[\beta(1-\delta)]^{i}}
$$

On détermine alors les seuils d'équilibre suivants :

$\Delta \tilde{a}^{T-1}=\gamma_{f}$

$\Delta \tilde{a}^{T-2}\left[\left\{\sum_{i=0}^{1}[\beta(1-\delta)]^{i}\right\}\right]=\gamma_{f}$

$\Delta \tilde{a}^{T-3}\left[\left\{\sum_{i=0}^{2}[\beta(1-\delta)]^{i}\right\}+\alpha \beta^{2} \delta p^{T-2}(a)\right]=\gamma_{f}-\left(\tilde{a}^{T-3}-b\right) \alpha \beta^{2} \delta\left[p^{T-2}(a)-p_{0}^{T-2}(a)\right]$

$\Delta \tilde{a}^{T-4}\left[\left\{\sum_{i=0}^{3}[\beta(1-\delta)]^{i}\right\}+\alpha \beta^{2} \delta p^{T-3}(a)+\alpha \beta^{3}(1-\delta) \delta\left[p^{T-2}(a)+p^{T-3}(a)\right]+\beta^{3} \delta\left[1-p^{T-3}(a)\right] p^{T-2}(a)(1-\pi)\right]$

$=\gamma_{f}-\left(\tilde{a}^{T-4}-b\right) \alpha \beta^{2} \delta\left[\left[p^{T-3}(a)-p_{0}^{T-3}(a)\right]\left\{\sum_{i=0}^{1}[\beta(1-\delta)]^{i}\right\}+\beta(1-\delta)\left[p^{T-2}(a)-p_{0}^{T-2}(a)\right]\right.$

$\left.-\alpha \beta\left[p^{T-3}(a) p^{T-2}(a)-p_{0}^{T-3}(a) p_{0}^{T-2}(a)\right]+\beta^{2}\left[p^{T-2}(a)-p_{0}^{T-2}(a)\right]\left[1-\pi\left[1-p^{T-3}(a)\right]\right]\right]$

\section{Seuils de formation à l'optimum}

Le problème du planificateur consiste à choisir le seuil d'aptitude $a_{t}^{\star} \forall t$ qui vérifie $\tilde{Y}_{t}\left(a_{t}^{\star}\right)-$ $\gamma_{F}=\hat{Y}_{t}\left(a_{t}^{\star}\right)$.

On pose alors :

$(1+\Delta) a+\beta\left[(1-\delta) \tilde{Y}_{t+1}(a)+\delta Y_{t+1}^{u 2}(a)\right]-\gamma_{f}=a+\beta\left[(1-\delta) \hat{Y}_{t+1}(a)+\delta Y_{t+1}^{u 0}(a)\right]$ 
On obtient alors la condition suivante :

$$
\begin{aligned}
\Delta a_{t}^{\star}= & \gamma_{f}-\beta(1-\delta)\left[\tilde{Y}_{t+1}\left(a_{t}^{\star}\right)-\hat{Y}_{t+1}\left(a_{t}^{\star}\right)\right]-\beta \delta\left[Y_{t+1}^{u 2}\left(a_{t}^{\star}\right)-Y_{t+1}^{u 0}\left(a_{t}^{\star}\right)\right] \\
= & \gamma_{f}-\beta(1-\delta)\left[\left(\tilde{Y}_{t+1}\left(a_{t}^{\star}\right)-Y_{t+1}^{u 2}\left(a_{t}^{\star}\right)\right)-\left(\hat{Y}_{t+1}\left(a_{t}^{\star}\right)-Y_{t+1}^{u 0}\left(a_{t}^{\star}\right)\right)\right] \\
& -\beta\left[Y_{t+1}^{u 2}\left(a_{t}^{\star}\right)-Y_{t+1}^{u 0}\left(a_{t}^{\star}\right)\right]
\end{aligned}
$$

En utilisant les expressions des valeurs sociales, on montre que :

$$
\begin{aligned}
& \left(\tilde{Y}_{t+1}\left(a_{t}^{\star}\right)-Y_{t+1}^{u 2}\left(a_{t}^{\star}\right)\right)-\left(\hat{Y}_{t+1}\left(a_{t}^{\star}\right)-Y_{t+1}^{u 0}\left(a_{t}^{\star}\right)\right) \\
& =\Delta a_{t}^{\star}+\beta(1-\delta)\left[\left(\tilde{Y}_{t+2}\left(a_{t}^{\star}\right)-Y_{t+2}^{u 2}\left(a_{t}^{\star}\right)\right)-\left(\hat{Y}_{t+2}\left(a_{t}^{\star}\right)-Y_{t+2}^{u 0}\left(a_{t}^{\star}\right)\right)\right] \\
& \quad-\beta\left[p^{t+1}(a)\left(\tilde{Y}_{t+2}\left(a_{t}^{\star}\right)-Y_{t+2}^{u 2}\left(a_{t}^{\star}\right)\right)-p_{0}^{t+1}(a)\left(\hat{Y}_{t+2}\left(a_{t}^{\star}\right)-Y_{t+2}^{u 0}\left(a_{t}^{\star}\right)\right)\right] \\
& \quad+\beta \pi\left[1-p^{t+1}(a)\right]\left[Y_{t+2}^{u 2}\left(a_{t}^{\star}\right)-Y_{t+2}^{u 0}\left(a_{t}^{\star}\right)\right]
\end{aligned}
$$

On peut également montrer que :

$$
\begin{aligned}
Y_{t+1}^{u 2}\left(a_{t}^{\star}\right)-Y_{t+1}^{u 0}\left(a_{t}^{\star}\right)= & \beta\left[p^{t+1}(a)\left(\tilde{Y}_{t+2}\left(a_{t}^{\star}\right)-Y_{t+2}^{u 2}\left(a_{t}^{\star}\right)\right)-p_{0}^{t+1}(a)\left(\hat{Y}_{t+2}\left(a_{t}^{\star}\right)-Y_{t+2}^{u 0}\left(a_{t}^{\star}\right)\right)\right] \\
& +\beta\left[Y_{t+2}^{u 2}\left(a_{t}^{\star}\right)-Y_{t+2}^{u 0}\left(a_{t}^{\star}\right)\right]-\beta \pi\left[1-p^{t+1}(a)\right]\left[Y_{t+2}^{u 2}\left(a_{t}^{\star}\right)-Y_{t+2}^{u 0}\left(a_{t}^{\star}\right)\right]
\end{aligned}
$$

On remarque alors que :

$$
\begin{aligned}
& \left(\tilde{Y}_{t+1}\left(a_{t}^{\star}\right)-Y_{t+1}^{u 2}\left(a_{t}^{\star}\right)\right)-\left(\hat{Y}_{t+1}\left(a_{t}^{\star}\right)-Y_{t+1}^{u 0}\left(a_{t}^{\star}\right)\right) \\
& =\Delta a_{t}^{\star}+\beta(1-\delta)\left[\left(\tilde{Y}_{t+2}\left(a_{t}^{\star}\right)-Y_{t+2}^{u 2}\left(a_{t}^{\star}\right)\right)-\left(\hat{Y}_{t+2}\left(a_{t}^{\star}\right)-Y_{t+2}^{u 0}\left(a_{t}^{\star}\right)\right)\right] \\
& \quad-\left[Y_{t+1}^{u 2}\left(a_{t}^{\star}\right)-Y_{t+1}^{u 0}\left(a_{t}^{\star}\right)\right]+\beta\left[Y_{t+2}^{u 2}\left(a_{t}^{\star}\right)-Y_{t+2}^{u 0}\left(a_{t}^{\star}\right)\right]
\end{aligned}
$$


De la même façon qu'à l'équilibre, on montre par itération la condition suivante :

$$
\Delta a_{t}^{\star}=\frac{\gamma_{f}-\left\{\sum_{i=0}^{T-3-t} \beta \delta[\beta(1-\delta)]^{i}\left[Y_{t+1+i}^{u 2}\left(a_{t}^{\star}\right)-Y_{t++1+i}^{u 0}\left(a_{t}^{\star}\right)\right]\right\}}{\sum_{i=0}^{T-1-t}[\beta(1-\delta)]^{i}}
$$

On détermine alors les seuils optimaux suivants :

$\Delta a_{T-1}^{\star}=\gamma_{f}$

$\Delta a_{T-2}^{\star}\left[\left\{\sum_{i=0}^{1}[\beta(1-\delta)]^{i}\right\}\right]=\gamma_{f}$

$\Delta a_{T-3}^{\star}\left[\left\{\sum_{i=0}^{2}[\beta(1-\delta)]^{i}\right\}+\beta^{2} \delta p^{T-2}(a)\right]=\gamma_{f}-\left(a_{T-3}^{\star}-b\right) \beta^{2} \delta\left[p^{T-2}(a)-p_{0}^{T-2}(a)\right]$

$\Delta a_{T-4}^{\star}\left[\left\{\sum_{i=0}^{3}[\beta(1-\delta)]^{i}\right\}+\beta^{2} \delta p^{T-3}(a)+\beta^{3}(1-\delta) \delta\left[p^{T-2}(a)+p^{T-3}(a)\right]+\beta^{3} \delta\left[1-p^{T-3}(a)\right] p^{T-2}(a)(1-\pi)\right]$

$=\gamma_{f}-\left(a_{T-4}^{\star}-b\right) \beta^{2} \delta\left[\left[p^{T-3}(a)-p_{0}^{T-3}(a)\right]\left\{\sum_{i=0}^{1}[\beta(1-\delta)]^{i}\right\}+\beta(1-\delta)\left[p^{T-2}(a)-p_{0}^{T-2}(a)\right]\right.$

$\left.-\beta\left[p^{T-3}(a) p^{T-2}(a)-p_{0}^{T-3}(a) p_{0}^{T-2}(a)\right]+\beta^{2}\left[p^{T-2}(a)-p_{0}^{T-2}(a)\right]\left[1-\pi\left[1-p^{T-3}(a)\right]\right]\right]$ 
14-5. La persistance du chômage ultra-marin

Yannick L'Horty

14-4. Grèves et productivité du travail : Application au cas français Jérémy Tanguy

14-3. Le non-recours au RSA "socle seul": L'hypothèse du patrimoine Sylvain Chareyron

14-2. Une évaluation de l'impact de l'aménagement des conditions de travail sur la reprise du travail après un cancer

Emmanuel Duguet, Christine Le Clainche

14-1. Renforcer la progressivité des prélèvements sociaux

Yannick L'Horty, Etienne Lehmann

\section{TEPP Rapports de Recherche 2013}

13-10. La discrimination à l'entrée des établissements scolaires privés : Les résultats d'une expérience contrôlée

Loïc du Parquet, Thomas Brodaty, Pascale Petit

13-9. Simuler les politiques locales favorisant l'accessibilité à l'emploi

Mathieu Bunel, Elisabeth Tovar

13-8. Le paradoxe des nouvelles politiques d'insertion

Jekaterina Dmitrijeva, Florent Fremigacci, Yannick L'Horty

13-7. L'emploi des seniors : un réexamen des écarts de taux d'emploi européens Laetitia Challe

13-6. Effets de quartier, effet de département : discrimination liée au lieu de résidence et accès à l'emploi

Pascale Petit, Mathieu Bunel, Emilia Ene Jones, Yannick L'Horty

13-5. Comment améliorer la qualité des emplois salariés exercés par les étudiants ? Les enseignements d'une expérience contrôlée

Jekaterina Dmitrijeva, Yannick L'Horty, Loïc Du Parquet, Pascale Petit

13-4. Evaluer l'efficacité d'une campagne de valorisation du bénévolat : Les enseignements de deux expériences contrôlées sur le marché du travail

Thomas Brodaty, Céline Emond, Yannick L'Horty, Loïc Du Parquet, Pascale Petit

13-3. Les différents parcours offerts par l'Education Nationale procurent-ils les mêmes chances d'accéder à l'emploi ?

Florent Fremigacci, Yannick L’Horty, Loïc Fu Parquet, Pascale Petit

13-2. Faut-il subventionner le permis de conduire des jeunes en difficulté d'insertion ?

Yannick L’Horty, Emmanuel Duguet, Pascale Petit, Bénédicte Rouland, Yiyi Tao 
La Fédération de recherche CNRS Travail, Emploi et Politiques Publiques (TEPP, FR $\mathbf{n}^{\circ} \mathbf{3 4 3 5}$ ) réunit des centres de recherche en économie et sociologie :

- Le Centre d'Etudes des Politiques Economiques de l'université d'Evry, EPEE, Université d'Evry Val d'Essonne

- Le Centre Pierre Naville, CPN, Université d'Evry Val d'Essonne

- Le Centre de Recherche en Economie et Management, CREM, Université de Caen Basse Normandie et Université de Rennes 1

- L'Equipe de Recherche sur les Marchés, l'Emploi et la Simulation, ERMES, Université deParis II Panthéon-Assas

- L'Equipe de Recherche sur l'Utilisation des Données Temporelles en Economie, ERUDITE, Université de Paris-Est Créteil et Université de Paris-Est Marne-la-Vallée

- Le Groupe d'Analyse des Itinéraires et des Niveaux Salariaux, GAINS, Université du Maine

La Fédération TEPP rassemble 150 chercheurs et enseignants-chercheurs, 140 doctorants et 40 chercheurs associés, qui étudient les mutations du travail et de l'emploi en relation avec les choix des entreprises et analysent les politiques publiques en mobilisant les nouvelles méthodes d'évaluation. 